\title{
A new prognostic model for hepatocellular carcinoma recurrence after curative hepatectomy
}

\author{
YUKIO TOKUMITSU $^{1}$, KAZUHIKO SAKAMOTO ${ }^{1}$, YOSHIHIRO TOKUHISA ${ }^{1}$, \\ HIROTO MATSUI $^{1}$, SATOSHI MATSUKUMA $^{1}$, YOSHINARI MAEDA ${ }^{2}$, KOICHIRO SAKATA $^{3}$, \\ HIROSHI WADA ${ }^{4}$, HIDETOSHI EGUCHI ${ }^{4}$, HIROYUKI OGIHARA ${ }^{5}$, YUSUKE FUJITA ${ }^{6}$, \\ YOSHIHIKO HAMAMOTO ${ }^{6}$, NORIO IIZUKA ${ }^{7},{\text { TOMIO } \text { UENO }^{8} \text { and HIROAKI NAGANO }}^{1}$
}

${ }^{1}$ Department of Gastroenterological, Breast and Endocrine Surgery,

Yamaguchi University Graduate School of Medicine, Ube, Yamaguchi 755-8505; ${ }^{2}$ Department of Surgery, Kanmon Medical Center, National Hospital Organization, Shimonoseki, Yamaguchi 752-8510; ${ }^{3}$ Department of Surgery, Shimonoseki Medical Center, Japan Community Health Care Organization, Shimonoseki, Yamaguchi 750-0061;

${ }^{4}$ Department of Gastroenterological Surgery, Osaka University Graduate School of Medicine, Suita, Osaka 565-0871;

${ }^{5}$ Department of Biomolecular Engineering Applied Molecular Bioscience, Yamaguchi University Graduate School of

Medicine; ${ }^{6}$ Department of Information Science and Engineering, Yamaguchi University Graduate School

of Sciences and Technology for Innovation, Ube, Yamaguchi 755-8611; ${ }^{7}$ Department of Kampo Medicine, Graduate School of Biomedical and Health Sciences, Hiroshima University, Hiroshima 734-8553; ${ }^{8}$ Department of Digestive Surgery, Kawasaki Medical School, Kurashiki, Okayama 701-0192, Japan

Received August 8, 2017; Accepted October 10, 2017

DOI: $10.3892 / \mathrm{ol} .2018 .7821$

\begin{abstract}
We previously reported the effectiveness of the product of tumor number and size ( $\mathrm{NxS}$ factor) for the prognosis of hepatocellular carcinoma (HCC) in patients following hepatectomy. The present study aimed to propose a new score based on the NxS factor to predict HCC recurrence following hepatectomy. A total of 406 patients who underwent hepatectomy for HCC at Osaka University Graduate School of Medicine were retrospectively analyzed to develop the new score. Among clinicopathological factors, including the NxS factor, the marker subset that achieved the best performance for prediction of early recurrence was assessed, and a prognostic model for HCC recurrence after curative hepatectomy (REACH) was developed. As the validation set, 425 patients who underwent hepatectomy for HCC at Yamaguchi University Graduate School of Medicine and Shimonoseki Medical Center were analyzed, and the prognostic ability of the REACH score was compared with that of well-known staging systems. Following analysis, the REACH score was
\end{abstract}

Correspondence to: Dr Yukio Tokumitsu, Department of Gastroenterological, Breast and Endocrine Surgery, Yamaguchi University Graduate School of Medicine, 1-1-1 Minamikogushi, Ube, Yamaguchi 755-8505, Japan

E-mail: yukio790604@gmail.com

Key words: prognostic score, hepatocellular carcinoma, recurrence, hepatectomy constructed using six covariates ( $\mathrm{NxS}$ factor, microscopic hepatic vein invasion, differentiation, serum albumin, platelet count and indocyanine green retention rate at $15 \mathrm{~min}$ ). In the validation set, the REACH score predicted early recurrence in 73 of 81 samples, with a sensitivity of $89 \%$ and a specificity of $58 \%$. The area under the curve (AUC) of the receiver operating characteristic curve of the REACH score was 0.78 and 0.74 , respectively, for 1- and 2-year recurrence after hepatectomy; each AUC was higher than that of any of the other staging systems. Survival analysis indicated the REACH score had the best predictive value in disease-free and overall survival. The present findings demonstrated that the REACH score may be used to classify patients with HCC into high- and low-risk of recurrence, and to predict subsequent survival following hepatic resection.

\section{Introduction}

Hepatocellular carcinoma (HCC) is one of the most common malignancies worldwide, with the prognosis highly dependent on tumor extension and liver function (1).

Although resection provides one of the best chances for a cure in patients with HCC, the majority of patients with HCC who undergo complete tumor resection subsequently develop tumor recurrence. Therefore, HCC continues to be a major cause of death $(2,3)$. Because of the high recurrence rate and poor prognosis, prognostic assessment and selection of treatment strategy in $\mathrm{HCC}$ patients are quite important. With the emergence of effective systemic chemotherapies (4) and antiviral agents (5), a precise predictive system for HCC patients is required. 
It has been generally known that early recurrence of HCC is the major risk factor affecting survival after hepatic resection. The identification of patients at high or low risk for recurrence after curative hepatectomy would help determine additional therapeutic and management strategies in clinical practice. During the last two decades, several prognostic staging systems based on clinicopathological data have been proposed for HCC, such as the Japan Integrated Staging (JIS) score $(6,7)$, the modified JIS score $(8,9)$, the Cancer of the Liver Italian Program (CLIP) score $(10,11)$, the Tokyo Score (12), and the Barcelona Clinic Liver Cancer (BCLC) staging system $(13,14)$. Although these scoring systems serve to classify patients into various groups with varying outcomes, they were not developed for use in predicting recurrence after surgical resection. Thus, we believe there is a need for an accurate model based on clinicopathological data for predicting the probability of $\mathrm{HCC}$ recurrence after curative liver resection.

It has been generally confirmed that certain clinicopathological data including tumor multiplicity, large tumor size and vascular invasion are poor prognostic indicators and risk factors for early recurrence of HCC (15-19). Previously, we reported that the mathematical product of tumor number and size of largest tumor ( $\mathrm{NxS}$ factor) could be a comprehensive measure of tumor burden to predict the prognosis of HCC patients who underwent liver resection, especially for predicting disease-free survival $(20,21)$. The aim of the present study was to develop a simple and practical model to discriminate early recurrence risk based on the $\mathrm{NxS}$ factor in conjunction with particular pathological findings or cirrhosis-associated parameters. The present study also compared the predictive accuracy between the NxS factor-based staging system and the currently used staging systems (JIS, modified JIS, CLIP, Tokyo score, BCLC) to determine the most appropriate prognostic model for $\mathrm{HCC}$ recurrence.

\section{Materials and methods}

Patients. The subjects selected for training analysis were the patients who underwent hepatectomy for HCC at the Department of Gastroenterological Surgery, Osaka University Graduate School of Medicine. Between May 1992 and December 2008, a total of 578 patients with HCC underwent hepatectomy. The diagnoses of HCC were all confirmed pathologically. We defined curative hepatectomy as complete resection of all tumor nodules without involving any major branch of the portal or hepatic veins. Patients were excluded from the analysis if they met any of the following criteria: volume reduction surgery, hepatectomy with intraoperative ablation therapy, HCC with major branch of portal or hepatic vein thrombosis, death within 30 days after surgery, death from other disease, and insufficient clinical data. Finally, 406 patients were enrolled in the study as the training set. All patients were followed up after hepatectomy until death or the date of the last follow-up visit, and survival was censored in December 2012.

The subjects selected for validation analysis were 357 patients who underwent curative hepatectomy for HCC at the Department of Gastroenterological, Breast and Endocrine Surgery, Yamaguchi University Graduate School of Medicine from January 1985 to December 2012, and 68 patients who underwent curative hepatectomy for HCC at Shimonoseki Medical Center between March 2007 and December 2012. Inclusion and exclusion criterion for participation in the study were the same as for the training set. All patients were followed up after hepatectomy until death or the date of the last follow-up visit, and survival was censored in December 2015.

Data on tumor factors such as maximum size of the main tumor, number of tumors, differentiation, and microvascular invasion were based on the final pathological findings of the resected liver. Laboratory data, including serum bilirubin, albumin, prothrombin activity, platelet count, indocyanine green retention rate at $15 \mathrm{~min}$ (ICG-R15), hepatitis B surface antigen, anti-hepatitis $\mathrm{C}$ antibody, and $\alpha$-fetoprotein (AFP) were obtained before operation. The Child-Pugh classification (22), the degree of liver damage classification by the Liver Cancer Study Group of Japan (LCSGJ) (23), TNM staging system (LCSGJ), TNM staging system (Union for International Cancer Control, UICC), JIS score, modified JIS score, CLIP score, Tokyo score and BCLC staging system were evaluated using these variables.

The collected data were statistically analyzed and assessed at Yamaguchi University. Each institution obtained institutional review board approval.

Methods. The novel predictive system with predictive markers and optimal cut-off point was established using the training set. The score point (SP) of each marker was decided in advance. The total score point (TSP) was defined as the summation of the SP for each predictive marker used. If the TSP was less than or equal to the previously defined cut-off point, the patient was classified into the low risk group for early recurrence. Otherwise, the patient was classified into the high risk group for early recurrence. To predict the probability of early HCC recurrence at one year after hepatectomy, we found the subset of predictive markers and the optimal cut-off point that maximized the sensitivity under the constrained conditions of a specificity $\geqq 50 \%$. Finally, we determined an optimal predictive model using the subset of selected predictive markers and the optimal cut-off point.

Algorithm for selection of the predictive markers and the optimal cut-off point. $\mathrm{M}$ represents the number of candidate markers. The marker selection problem is to select a predictive marker subset of size $d$ from the marker set of M candidate markers. The initial value of $d$ was set as three.

Step 1. For a marker subset of size d, the sensitivity and specificity were calculated throughout all expected cut-off points of the TSP. For the marker subset of size d, the optimal cut off point of the TSP, which had the highest sensitivity with the specificity $\geqq 50 \%$ was found. Note that any marker subset of size d with the specificity $<50 \%$ was omitted.

Step 2. For all possible maker subsets of size d, Step 1 was repeated.

Step 3. The marker subset, which maximized the sensitivity, was selected from maker subsets of size d with the optimal cut off point of the TSP. 
Table I. Patient profiles and tumor characteristics.

Number of patients

Variables

Training set $(n=406)$

Validation set $(n=425)$

P-value

Host factors

Age

Mean \pm standard error (years)

$65.0 \pm 0.44$

$65.2 \pm 0.45$

0.7509

Sex

Male/female

$322 / 84$

$321 / 104$

0.1929

HBs Ag

Positive/negative

$77 / 329$

$80 / 345$

0.9583

$\mathrm{HCV} \mathrm{Ab}$

Positive/negative

$243 / 163$

252/173

0.8698

Bilirubin (mg/dl)

$<1 / \geq 1$

$337 / 69$

$303 / 122$

0.0001

Albumin (g/dl)

$\leq 3.5 />3.5$

$158 / 248$

$134 / 291$

0.0258

Prothrombin time (\%)

$<80 / \geq 80$

261/145

$121 / 304$

$<0.0001$

ICGR-15 (\%)

$<15 / \geq 15$

207/199

$218 / 207$

0.929

Platelet count $\left(\mathrm{x} 10^{4} / \mathrm{mm}^{3}\right)$

$<10 / \geq 10$

$95 / 311$

$98 / 327$

0.9076

Degree of liver damage

$\mathrm{A} / \mathrm{B}$

$224 / 182$

$288 / 137$

0.0002

Child-Pugh classification

A/B

$336 / 70$

$390 / 35$

0.0001

Tumor factors

Tumor size (maximum diameter) $(\mathrm{cm})$

$<2 / \geq 2,<5 / \geq 5$

$93 / 220 / 93$

$90 / 262 / 73$

0.0582

Number of tumors

$1 / 2-3 / \geq 4$

$288 / 103 / 15$

$303 / 96 / 26$

0.2075

The NxS factor

$<4 / 4-9 />9$

$215 / 128 / 63$

$228 / 130 / 67$

0.9581

Macroscopic portal vein invasion

Absent/present

$379 / 27$

$372 / 53$

0.0045

Macroscopic hepatic vein invasion

Absent/present

$406 / 0$

$405 / 20$

$<0.0001$

Microscopic portal vein invasion

Absent/present

$302 / 104$

$324 / 101$

0.5361

Microscopic hepatic vein invasion

Absent/present

$406 / 0$

$331 / 94$

$<0.0001$

$\operatorname{AFP}(\mathrm{ng} / \mathrm{ml})$

$<400 / \geq 400$

$332 / 74$

$345 / 80$

0.8248

Differentiation

Well/moderate/poor

29/191/186

$97 / 274 / 54$

$<0.05$

Anatomical resection

Yes/no

$157 / 249$

274/151

$<0.0001$

Staging systems

TNM stage (LCSGJ)

I/II/III/IV 
Table I. Continued.

\begin{tabular}{|c|c|c|c|}
\hline \multirow[b]{2}{*}{ Variable } & \multicolumn{2}{|c|}{ Number of patients } & \multirow[b]{2}{*}{ P-value } \\
\hline & Training set $(n=406)$ & Validation set $(n=425)$ & \\
\hline \multicolumn{4}{|c|}{ TNM stage (UICC) } \\
\hline I/II/III/IV & $221 / 149 / 33 / 3$ & $205 / 184 / 33 / 3$ & 0.2784 \\
\hline \multicolumn{4}{|l|}{ JIS score } \\
\hline $0 / 1 / 2 / 3 / 4$ & 67/149/133/49/8 & $51 / 169 / 141 / 61 / 3$ & 0.1462 \\
\hline \multicolumn{4}{|c|}{ Modified JIS score } \\
\hline $0 / 1 / 2 / 3 / 4$ & $49 / 123 / 135 / 83 / 16$ & $41 / 131 / 158 / 79 / 16$ & 0.6565 \\
\hline \multicolumn{4}{|l|}{ Tokyo score } \\
\hline $0 / 1 / 2 / 3 / 4-6$ & $48 / 138 / 155 / 57 / 8$ & 38/160/137/60/30 & 0.0027 \\
\hline \multicolumn{4}{|l|}{ CLIP score } \\
\hline $0 / 1 / 2 / 3-5$ & $179 / 140 / 50 / 37$ & $221 / 132 / 52 / 20$ & 0.0253 \\
\hline \multicolumn{4}{|c|}{ BCLC staging system } \\
\hline 0/A/B/C & $63 / 257 / 57 / 29$ & $36 / 281 / 61 / 47$ & 0.0061 \\
\hline
\end{tabular}

Step 4 . We incremented the previous number $\mathrm{d}$ by $1(\mathrm{~d} \leftarrow \mathrm{d}+1)$. When $\mathrm{d}<\mathrm{M}$, we repeated from Step 1 to Step 3. When $\mathrm{d}=\mathrm{M}$, we stopped the maker selection and obtained the M-3 marker subsets with optimal cut-off point.

Step 5. Finally, we determined the best maker subset which had the highest sensitivity with smaller number of markers among the M-3 marker subsets obtained.

Statistical analysis. We used the Chi-square test, Fisher's exact test, and Student's t-test to assess differences in two or three categorical variables. To evaluate the prognostic ability for 1-year recurrence, the area under the receiver operating characteristic (ROC) curve (AUC) for each staging system was calculated. Disease-free survival (DFS) and overall survival (OS) curves were plotted with the Kaplan-Meier method, and differences in DFS and OS between the groups were compared by using a log-rank test in univariate analysis. Staging systems for HCC were tested by univariate and multivariate Cox analyses in both DFS and OS. Statistical analysis was performed using JMP version 9.0 (SAS Institute Japan, Tokyo, Japan).

\section{Results}

Development of a novel predictive system. The baseline characteristics of the 406-member training set and 425-member validation set are shown in Table I. There were significant differences between the training and validation sets regarding serum bilirubin, albumin, prothrombin time, degree of liver damage, Child-Pugh classification, macroscopic portal vein invasion, macroscopic hepatic vein invasion, microscopic hepatic vein invasion, differentiation, and operation method (anatomical resection or not) $(\mathrm{P}<0.05)$.
It was considered that the main prognostic factors for HCC patients after hepatectomy were the TNM stage, vascular invasion, the number of tumors, histological differentiation and liver function $(2,24)$. Thus, we chose nine candidates including $\mathrm{NxS}$ factor which constituted aforementioned factors. Univariate analysis of the nine candidate markers in the training set are shown in Table II. There were significant differences between the early recurrence group and no recurrence group regarding the product of tumor number and size (NxS), microscopic portal vein invasion and differentiation (well- and moderately-differentiated HCC vs. poorly-differentiated HCC). These variables had the following effects in determining the final score. Table II shows the SP of each candidate marker equivalent to the marker's weight. For the NxS factor, zero points were assigned for $\mathrm{NxS}<4$, two points for $\mathrm{NxS}$ of 4 to 9 , and three points for NxS $>9$. For microscopic portal vein invasion, zero points were assigned for a portal vein invasion of negative, and two points for a portal vein invasion of positive. For microscopic hepatic vein invasion, zero points were assigned for a hepatic vein invasion of negative, and one point for a hepatic vein invasion of positive. For differentiation, zero points were assigned for well- or moderately-differentiated HCC, and one point for poorly-differentiated HCC. For other liver function data, zero points were assigned for good liver function level (bilirubin $<1$, albumin $>3.5$, prothrombin time $\geq 80$, ICG-R15 $<15$, and platelet count $\geq 10$ ), and one point for poor liver function (bilirubin $\geq 1$, albumin $\leq 3.5$, prothrombin time $<80$, ICG-R15 $\geq 15$ and platelet count $<10$ ).

The novel predictive system was established using predictive markers selected from the aforementioned candidate markers in the training set. The appropriate models and their optimal cut-off points for definition of early recurrence of each 
Table II. Univariate analysis and score point of the candidate markers in training set $(n=406)$.

\begin{tabular}{|c|c|c|c|c|}
\hline Variables & $\begin{array}{c}\text { ER } \\
(n=155)\end{array}$ & $\begin{array}{c}\text { NER } \\
(n=251)\end{array}$ & P-value & $\begin{array}{c}\text { Score } \\
\text { point (SP) }\end{array}$ \\
\hline The NxS factor & & & $<0.0001$ & \\
\hline$<4$ & 64 & 151 & & 0 \\
\hline $4-9$ & 50 & 78 & & 2 \\
\hline$>9$ & 41 & 22 & & 3 \\
\hline \multicolumn{5}{|l|}{$\begin{array}{l}\text { Microscopic portal } \\
\text { vein invasion }\end{array}$} \\
\hline$(-)$ & 91 & 211 & & 0 \\
\hline$(+)$ & 64 & 40 & & 2 \\
\hline $\begin{array}{l}\text { Microscopic hepatic } \\
\text { vein invasion }\end{array}$ & & & - & \\
\hline$(-)$ & 155 & 251 & & 0 \\
\hline$(+)$ & 0 & 0 & & 1 \\
\hline Differentiation & & & $<0.0001$ & \\
\hline Well/moderate & 58 & 162 & & 0 \\
\hline Poor & 97 & 89 & & 1 \\
\hline Bilirubin (mg/dl) & & & 0.0845 & \\
\hline$<1$ & 135 & 202 & & 0 \\
\hline$\geq 1$ & 20 & 49 & & 1 \\
\hline Albumin (g/dl) & & & 0.1616 & \\
\hline$>3.5$ & 88 & 160 & & 0 \\
\hline$\leq 3.5$ & 67 & 91 & & 1 \\
\hline Prothrombin time $(\%)$ & & & 0.7261 & \\
\hline$>80$ & 57 & 88 & & 0 \\
\hline$\leq 80$ & 98 & 163 & & 1 \\
\hline ICG-R15 (\%) & & & 0.4105 & \\
\hline$<15$ & 75 & 132 & & 0 \\
\hline$\geq 15$ & 80 & 119 & & 1 \\
\hline $\begin{array}{l}\text { Platelet count } \\
\left(\mathrm{x} 10^{4} / \mathrm{mm}^{3}\right)\end{array}$ & & & 0.4303 & \\
\hline$\geq 10$ & 122 & 189 & & 0 \\
\hline$<10$ & 33 & 62 & & 1 \\
\hline
\end{tabular}

ER, early recurrence; ICG-R15, indocyanine green retention rate at $15 \mathrm{~min}$; ER, early recurrence; NER, non early recurrence; $\mathrm{NxS}$, the product of number and size.

number of selected markers $(d)$ are shown in Table III. Finally, the six main prognostic factors (NxS factor, microscopic portal vein invasion, differentiation, albumin, ICG-R15 and platelet count) were selected because the model using these six factors had the highest sensitivity (0.79) and accuracy (0.62) with the smallest number of markers (Table III), and a novel predictive model for HCC recurrence after curative hepatectomy (REACH) score was established (Table IV).

Comparison of predictive ability of staging systems. In the validation set, the REACH score predicted early recurrence at 1 year after hepatectomy in 73 of 82 samples and had a sensitivity of $89 \%$ and a specificity of $58 \%$. The REACH score had a higher sensitivity value with more than $50 \%$ specificity, and the highest Youden's index for recurrence within 1 year after hepatectomy than any cut-off points of the five clinical staging systems (JIS, modified JIS, Tokyo score, CLIP, and BCLC) (Table V). The AUCs of the ROCs of the REACH scores for 1- and 2-year recurrence after hepatectomy were 0.78 and 0.74 , respectively, and both of them were higher than those of any of the other staging systems. The Kaplan-Meier method estimated the DFS curves and OS curves according to the REACH score, JIS score, modified JIS score, Tokyo score, CLIP score, and BCLC staging system; these are shown in Figs. 1 and 2, respectively. In all scoring systems, there were significant differences $(\mathrm{P}<0.05)$ in both the DFS and OS between the high- and low-risk groups. Table VI displays the univariate and multivariate survival analyses using Cox's regression model for the optimal cut-off values of the REACH score and the known staging systems. In the univariate analysis, the REACH score demonstrated the highest hazard ratio of any of the other staging systems in both DFS (hazard ratio (HR) 2.84, 95\% confidence interval (CI: 2.22-3.65) and OS (HR, 3.03, 95\% CI: 2.18-4.26). In the multivariate analysis, the REACH score and the Tokyo score were independent prognostic factors in both DFS and OS. The REACH score demonstrated a higher HR than the Tokyo score for both DFS (HR 20.4, 95\% CI: 1.52-2.74) and OS (HR 3.03, 95\% CI: 1.48-3.18).

\section{Discussion}

The major finding of the present study was that early recurrence in patients who have undergone curative hepatectomy for HCC can be predicted accurately using our novel scoring system based on the mathematical product of the tumor number and size of the largest tumor. We previously reported that the NxS factor was a useful tumor factor to predict HCC recurrence after curative hepatectomy $(20,21)$. The optimal cut-off values of the NxS factor were determined to be 4 and 9 in reference to the Milan criteria (single tumor $\leq 5 \mathrm{~cm}$ in size or $\leq 3$ tumors each $\leq 3 \mathrm{~cm}$ in size); (25) and multivariate analysis (Cox proportional hazards model) for DFS revealed that the NxS factor was an independent predictor in HCC patients who underwent curative hepatectomy (20). Although both tumor number and size of the largest tumor are also used as the main parameters of the TNM staging system $(1,26)$, we reported that the score based on the NxS factor better predicted the prognosis of HCC patients than the TNM and TNM-based systems such as the JIS score and modified JIS score. Since we had already successfully transformed the factors of tumor number and largest tumor size into one single parameter, we decided to integrate the $\mathrm{NxS}$ factor into the novel score for prediction of HCC recurrence. The current study succeeded in developing a prognostic model based on the $\mathrm{NxS}$ factor, and showed that the REACH score was more predictive of early HCC recurrence than the known predictive systems of the JIS, modified JIS, Tokyo score, CLIP and BCLC staging system.

To provide more personalized therapeutic options for patients at high risk for early recurrence and to avoid unnecessary overtreatment for patients who have been cured by surgery alone, early recurrence or non-recurrence should 
Table III. The best combination of the candidate markers in the training set.

d

\begin{tabular}{|c|c|c|c|c|c|c|}
\hline \multirow[b]{2}{*}{ Candidate markers } & & & & & & \\
\hline & 3 & 4 & 5 & $6^{\mathrm{a}}$ & 7 & 8 \\
\hline The NxS factor & $\bullet$ & $\bullet$ & $\bullet$ & $\bullet$ & $\bullet$ & \\
\hline Microscopic portal vein invasion & & $\bullet$ & $\bullet$ & $\bullet$ & $\bullet$ & $\bullet$ \\
\hline Microscopic hepatic vein invasion & & & & & $\bullet$ & $\bullet$ \\
\hline Differentiation & $\bullet$ & - & $\bullet$ & $\bullet$ & $\bullet$ & $\bullet$ \\
\hline Bilirubin (mg/dl) & & - & & & & - \\
\hline Albumin $(\mathrm{g} / \mathrm{dl})$ & & & & $\bullet$ & $\bullet$ & $\bullet$ \\
\hline Prothrombin time (\%) & & & $\bullet$ & & & $\bullet$ \\
\hline ICG-R15 (\%) & - & & $\bullet$ & $\bullet$ & - & $\bullet$ \\
\hline Platelet count $\left(\times 10^{4} / \mathrm{mm}^{3}\right)$ & & & & - & - & - \\
\hline Cut-off value (for early recurrence) & 2 & 2 & 3 & 3 & 3 & 3 \\
\hline Sensitivity $(\%)$ & 0.73 & 0.75 & 0.77 & 0.79 & 0.79 & 0.72 \\
\hline Specificity (\%) & 0.51 & 0.50 & 0.53 & 0.51 & 0.51 & 0.50 \\
\hline Accuracy $(\%)$ & 0.59 & 0.60 & 0.62 & 0.62 & 0.62 & 0.59 \\
\hline Youden's Index & 0.24 & 0.26 & 0.30 & 0.30 & 0.30 & 0.22 \\
\hline
\end{tabular}

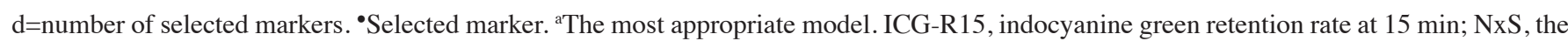
product of number and size.

be accurately predicted. Some well-known scoring systems for the estimation of prognostic outcomes in HCC such as the JIS, CLIP and BCLC have been developed to classify patients into various groups for risk stratification, and have been correlated with overall survival. However, these systems were not developed and validated for use in the prediction of early recurrence or after surgical resection. We previously reported that the predictive staging system based on the NxS factor has been developed to classify patients into 4 groups for risk stratification for disease free survival (20). Furthermore, in the current study, we successfully integrated the NxS factor into the REACH score to classify patients between high vs. low risk of recurrence following hepatic resection. Additionally, there is still no well-known predictive system based only on clinicopathological data for early recurrence. Although some nomogram-based systems using clinicopathological data have been proposed for predicting HCC recurrence (27-30), their accuracy has been limited by the precision of physical markings. Therefore, these prediction systems have not been externally validated and have not been widely used. We believed that an accurate model for predicting $\mathrm{HCC}$ recurrence after curative liver resection would help determine whether closer postoperative surveillance is needed, and additional therapeutic strategies such as salvage liver transplantations for recurrent HCC (31) could be applicable.

In recent years, molecular research has identified various biomarkers as predictive markers for $\mathrm{HCC}$ metastatic recurrence and clinical outcomes; and some molecular-based prognostic models have been developed (32-36). Kurokawa et al (34) reported a prediction model for HCC recurrence using a small-scale PCR-array system. Iizuka et al $(32,33)$ showed a correlation between
Table IV. The REACH score.

\begin{tabular}{lr}
\hline Variables & Point \\
\hline The NxS factor & \\
$<4$ & 0 \\
$4-9$ & 2 \\
$>9$ & 3
\end{tabular}

Microscopic portal vein invasion

$(+)$

$(-)$

Differentiation

Well/moderate $\quad 0$

Poor 1

Albumin (g/dl)

$>3.5$

0

$\leq 3.5$

ICG-R15 (\%)

$<15$

0

$\geq 15$

Platelet count $\left(\times 10^{4} / \mathrm{mm}^{3}\right)$

$\geq 10$

0

$<10$

1

ICG-R15, indocyanine green retention rate at $15 \mathrm{~min} ; \mathrm{NxS}$, the product of number and size; REACH, recurrence after curative hepatectomy.

gene expression and early (within 1 year) post-hepatectomy intrahepatic recurrence using a predictive system 
Table V. Probability of early recurrence according to the REACH score and known staging systems.

\begin{tabular}{|c|c|c|c|c|c|c|c|c|}
\hline \multirow[b]{2}{*}{ Model } & \multirow[b]{2}{*}{$\mathrm{n}$} & \multicolumn{5}{|c|}{ Probability of early recurrence (1-year) } & \multicolumn{2}{|c|}{$\begin{array}{l}\text { AUC for } \\
\text { recurrence }\end{array}$} \\
\hline & & ER/NER & P-value & Sensitivity & Specificity & Youden-index & 1-year & 2-year \\
\hline \multicolumn{9}{|c|}{ REACH score } \\
\hline$\leq 2$ & 209 & $9 / 200$ & $<0.0001$ & 0.89 & 0.58 & 0.47 & 0.78 & 0.75 \\
\hline$\geq 3$ & 216 & $73 / 143$ & & & & & & \\
\hline \multicolumn{9}{|l|}{ JIS score } \\
\hline 0 & 51 & $2 / 49$ & 0.0006 & 0.98 & 0.14 & 0.12 & 0.72 & 0.67 \\
\hline $1-4$ & 374 & $80 / 294$ & & & & & & \\
\hline 0,1 & 220 & $22 / 198$ & $<0.0001$ & 0.73 & 0.58 & 0.31 & & \\
\hline $2-4$ & 205 & $60 / 145$ & & & & & & \\
\hline $0-2$ & 361 & $48 / 313$ & $<0.0001$ & 0.42 & 0.91 & 0.33 & & \\
\hline 3,4 & 64 & $34 / 30$ & & & & & & \\
\hline $0-3$ & 422 & $79 / 343$ & 0.0004 & 0.04 & 1.00 & 0.04 & & \\
\hline 4 & 3 & $3 / 0$ & & & & & & \\
\hline \multicolumn{9}{|c|}{ Modified JIS score } \\
\hline 0 & 41 & $2 / 39$ & 0.0139 & 0.98 & 0.11 & 0.09 & 0.74 & 0.69 \\
\hline $1-4$ & 384 & $80 / 304$ & & & & & & \\
\hline 0,1 & 172 & $14 / 158$ & $<0.0001$ & 0.83 & 0.46 & 0.29 & & \\
\hline $2-4$ & 253 & $68 / 185$ & & & & & & \\
\hline $0-2$ & 330 & $37 / 293$ & $<0.0001$ & 0.55 & 0.85 & 0.40 & & \\
\hline 3,4 & 95 & $45 / 50$ & & & & & & \\
\hline $0-3$ & 409 & $71 / 338$ & $<0.0001$ & 0.13 & 0.99 & 0.12 & & \\
\hline 4 & 16 & $11 / 5$ & & & & & & \\
\hline \multicolumn{9}{|c|}{ Tokyo score } \\
\hline 0 & 38 & $5 / 33$ & 0.31 & 0.94 & 0.10 & 0.04 & 0.64 & 0.66 \\
\hline $1-6$ & 387 & $77 / 310$ & & & & & & \\
\hline 0,1 & 198 & $23 / 175$ & 0.0002 & 0.72 & 0.51 & 0.23 & & \\
\hline $2-6$ & 227 & $59 / 168$ & & & & & & \\
\hline $0-2$ & 335 & $53 / 282$ & 0.0008 & 0.35 & 0.82 & 0.18 & & \\
\hline $3-6$ & 90 & $29 / 61$ & & & & & & \\
\hline \multicolumn{9}{|c|}{ CLIP score } \\
\hline 0 & 269 & $33 / 236$ & $<0.0001$ & 0.60 & 0.69 & 0.29 & 0.67 & 0.61 \\
\hline $1-5$ & 156 & 49/107 & & & & & & \\
\hline 0,1 & 389 & $62 / 327$ & $<0.0001$ & 0.24 & 0.95 & 0.20 & & \\
\hline $2-5$ & 36 & $20 / 16$ & & & & & & \\
\hline \multicolumn{9}{|c|}{ BCLC staging system } \\
\hline 0 & 36 & $2 / 34$ & 0.0290 & 0.98 & 0.10 & 0.07 & 0.65 & 0.65 \\
\hline $\mathrm{A}, \mathrm{B}, \mathrm{C}$ & 389 & $80 / 309$ & & & & & & \\
\hline $0, \mathrm{~A}$ & 317 & $44 / 273$ & $<0.0001$ & 0.46 & 0.80 & 0.26 & & \\
\hline $\mathrm{B}, \mathrm{C}$ & 108 & $38 / 70$ & & & & & & \\
\hline $0, \mathrm{~A}, \mathrm{~B}$ & 377 & $64 / 313$ & 0.0007 & 0.22 & 0.91 & 0.13 & & \\
\hline $\mathrm{C}$ & 48 & $18 / 30$ & & & & & & \\
\hline
\end{tabular}

AUC, area under the curve; BCLC, Barcelona Clinic Liver Cancer; CLIP, Cancer of the Liver Italian Program; ER, early recurrence; JIS, Japan Integrated Staging; NER, non early recurrence; REACH, recurrence after curative hepatectomy.

consisting of 12 genes. Yoshioka et al (36) also reported a molecular-based diagnosis system for HCC recurrence using 172 informative genes. More recently, Srivastava et al (35) reported a morpho-molecular prognostic model based on the expression of selected proteins (p53, CD44, CD31, and AFP) and clinicopathological parameters, which could be used to classify HCC patients according to good vs. poor prognosis and high vs. low risk of recurrence following hepatic resection. 

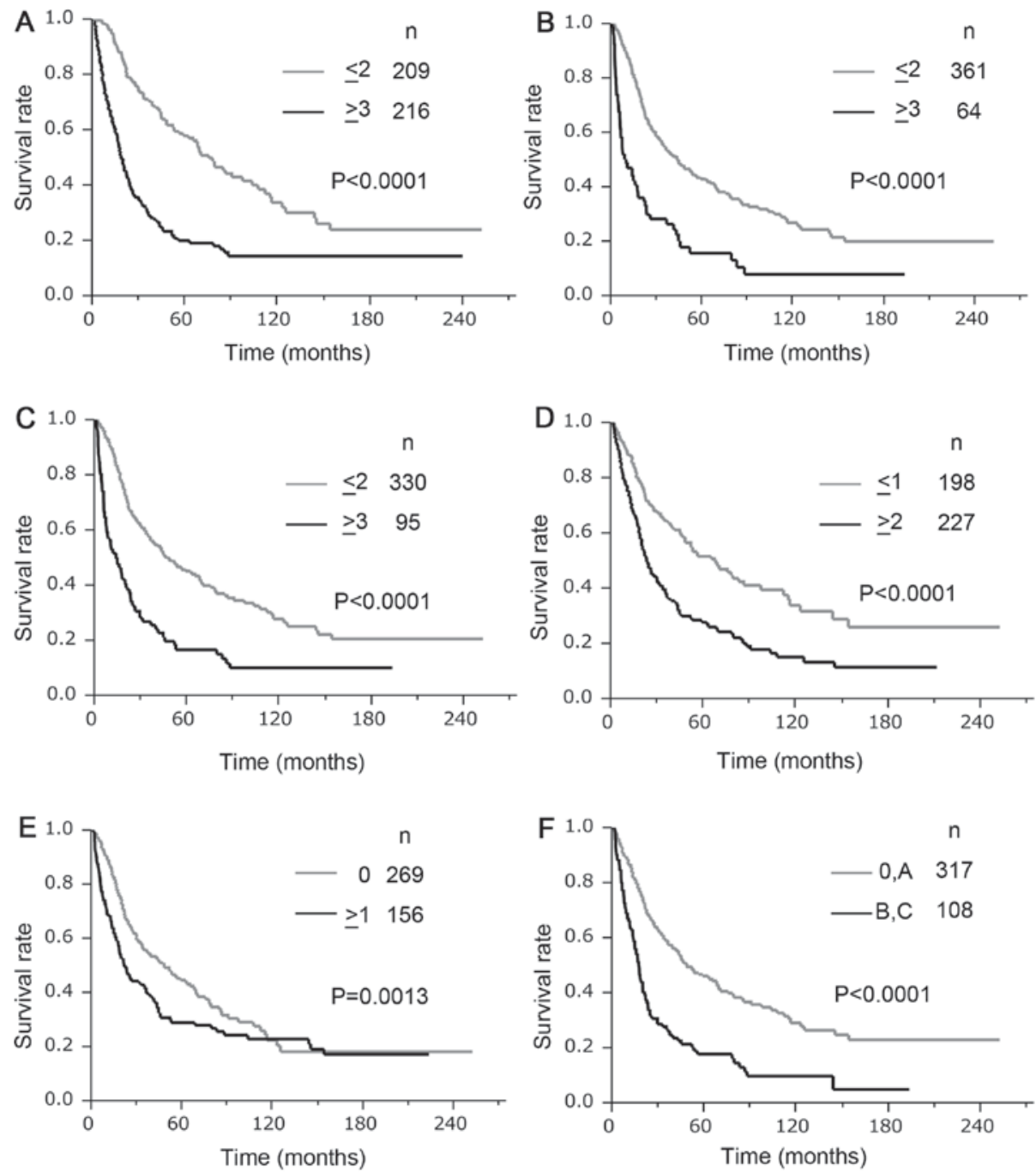

Figure 1. Comparison of disease-free survival according to scoring systems: (A) REACH score, (B) JIS score, (C) M-JIS score, (D) Tokyo score, (E) CLIP score, and (F) BCLC staging system. In all scoring systems, there were significant differences $(\mathrm{P}<0.05$, log-rank test) between the high- and low-risk groups. REACH, recurrence after curative hepatectomy; JIS, Japan Integrated Staging; M-JIS, modified Japan Integrated Staging; CLIP, Cancer of the Liver Italian Program; BCLC, Barcelona Clinic Liver Cancer.

Although these scoring systems have offered the possibility of high predictive ability for early recurrence, analysis of these genes in routine clinical practice is uncommon. The present study successfully demonstrated a new score based only on clinicopathological data, including the NxS factor, and provides a precise prediction of recurrence for patients who have undergone curative hepatectomy. Moreover, such a system can help determine optimal therapeutic and management strategies in daily practice, and act as the standard for comparison between molecular-based systems.

HCC recurrence derives from both residual intrahepatic metastasis (IM) and multicentric carcinogenesis (MC). Most late recurrence is considered to be MC, because MC depends not on the malignant potential of the resected HCC, but rather, on the degree of hepatitis or cirrhosis of the remnant liver (37-40). In contrast, it seems that IM is likely responsible for the majority of early recurrence within the first two postoperative years, because IM denotes the existence of non-visible intrahepatic metastasis at primary surgery $(39,40)$. Although the REACH score was developed to predict 1 -year recurrence after surgery to increase the power of sensitivity for early recurrence in the training set, we were able to show that the AUC values of the REACH score for both 1 and 2-year recurrence were higher than in any of the other systems used to analyze the validation set. Thus, the REACH score can be applicable to recurrence within two postoperative years, and can effectively detect residual metastasis.

For practical purposes, staging systems should be simple and based on data that are easily obtainable (12). One feature of the REACH score is that it consists of simple parameters (i.e., $\mathrm{NxS}$ factor, microscopic portal vein invasion, differentiation, albumin, ICG-R15 and platelet count) divided into two or three categories according to the predictive value of the HCC prognosis system. We examined only parameters that are easily obtainable, and we avoided criteria that are not generally available in daily practice. We also succeeded in creating an algorithm to develop the new simple prognostic scoring system, which consists of the best combination of the integer values assigned to each factor. We believe that the simplicity of a model is preferable for the sake of general versatility. Moreover, known prognostic scores have often been established by the selected 

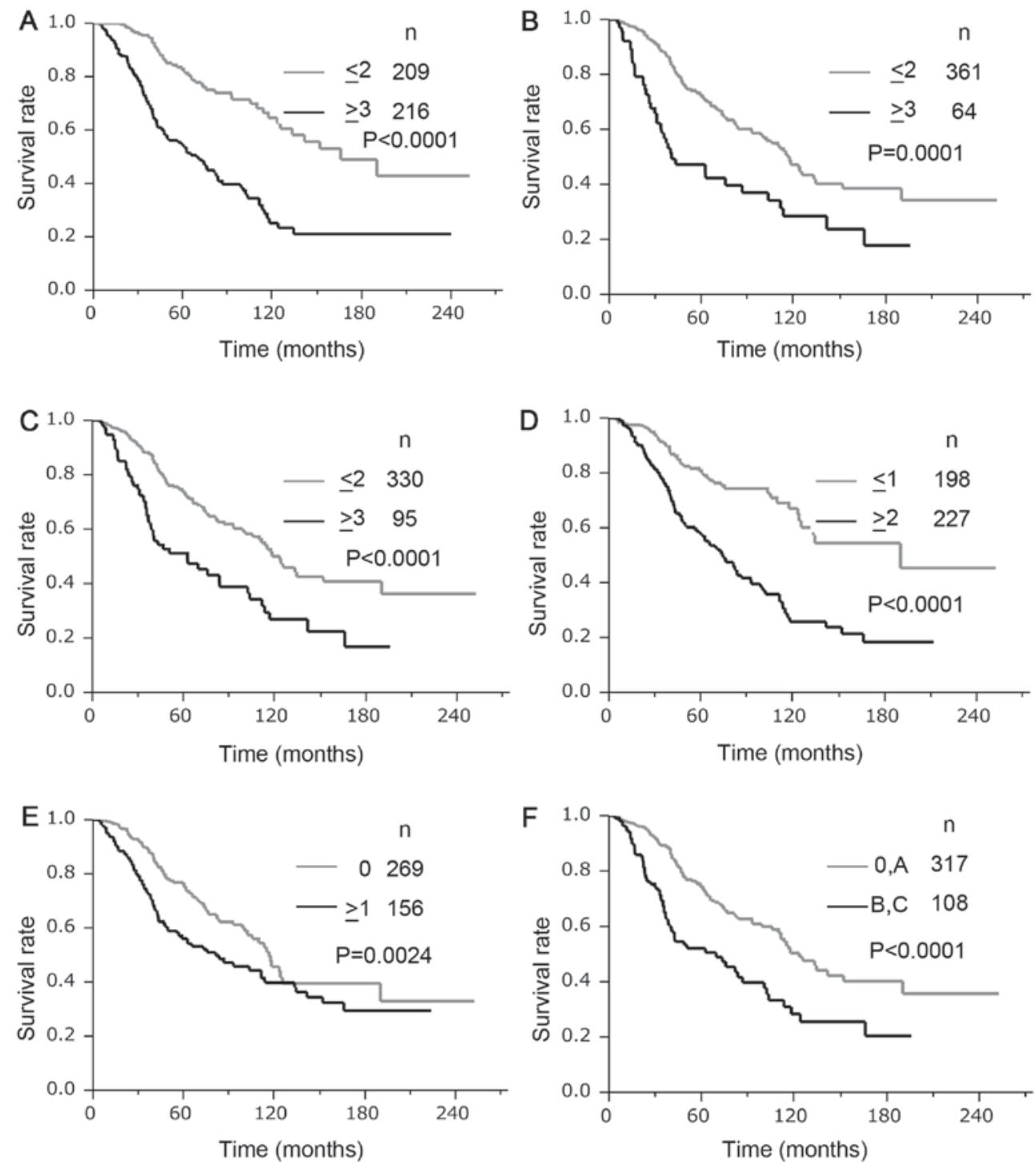

Figure 2. Comparison of overall survival according to scoring systems: (A) REACH score, (B) JIS score, (C) M-JIS score, (D) Tokyo score, (E) CLIP score, and (F) BCLC staging system. In all scoring systems, there were significant differences ( $\mathrm{P}<0.05$, log-rank test) between the high- and low-risk groups. REACH, recurrence after curative hepatectomy; JIS, Japan Integrated Staging; M-JIS, modified Japan Integrated Staging; CLIP, Cancer of the Liver Italian Program; BCLC, Barcelona Clinic Liver Cancer.

factors that showed statistical significance $(\mathrm{P}<0.05)$ as predictors (10-12), however, our comprehensive method of developing a new prognostic score could provide the prognostic combination of simple factors having clinical importance regardless of the level of statistical significance.

The present study had several limitations. First, we could find no existing well-known predictive systems based only on clinicopathological data for early recurrence as an appropriate model to compare with the REACH score. Thus, in the current study, we selected well-known clinical predictive systems for HCC prognosis to compare methods for assessing the REACH score. However, those other systems were originally developed to classify patients into various groups for risk stratification, not to classify patients between high vs. low risk of recurrence following hepatic resection. Second, the REACH score integrates the ICG-R15 test. Although ICG-R15 is an easily obtainable value which has been used routinely in the field of surgery in Japan and several Asian countries as a useful marker of hepatic function (41-43), and is also reported as a significant prognostic factor in HCC patients $(44,45)$, it is not routinely assessed worldwide. Thus, other classifications based on the well-known parameters such as the Child-Pugh classification (22) or albumin-bilirubin (ALBI) grade (46) may become alternative values of liver function in the prognostic score for the patients outside of Japan. Third, there is no standard of care for adjuvant therapy for HCC patients who underwent hepatectomy, thus, the REACH score may not provide valuable information to aid in the decision making regarding the application of adjuvant therapy at the current moment. However, the prognostic score based on the data of patients who did not receive effective adjuvant therapy will be valuable and act as the standard when the effective adjuvant therapies to prevent early $\mathrm{HCC}$ recurrence are developed, and we expect that the REACH score will help us better decide which patients need adjuvant therapy and which patients do not in the future. The fourth and fifth limitations of the present study were its retrospective nature, and the fact that it was based on only a Japanese population. Whether this score is applicable to other patient cohorts with different etiologic 
Table VI. Survival analysis of the REACH score and known staging systems.

A, Disease free survival

\begin{tabular}{|c|c|c|c|c|c|c|c|c|}
\hline \multirow[b]{2}{*}{ Model } & \multirow[b]{2}{*}{$\mathrm{n}$} & \multirow[b]{2}{*}{ MST (month) } & \multicolumn{3}{|c|}{ Univariate } & \multicolumn{3}{|c|}{ Multivariate } \\
\hline & & & $\mathrm{HR}$ & $95 \% \mathrm{CI}$ & P-value & $\mathrm{HR}$ & $95 \% \mathrm{CI}$ & P-value \\
\hline \multicolumn{9}{|c|}{ REACH score } \\
\hline$\leq 2$ & 209 & 75.8 & 1.00 & & & 1.00 & & \\
\hline$\geq 3$ & 216 & 18.6 & 2.84 & $2.22-3.65$ & $<0.0001$ & 2.04 & $1.52-2.74$ & $<0.0001$ \\
\hline \multicolumn{9}{|c|}{ JIS score } \\
\hline$\leq 2$ & 361 & 43.6 & 1.00 & & & & & \\
\hline$\geq 3$ & 64 & 9.7 & 2.56 & $1.89-3.42$ & $<0.0001$ & & & \\
\hline \multicolumn{9}{|c|}{ Modified JIS score } \\
\hline$\leq 2$ & 330 & 47.8 & 1.00 & & & & & \\
\hline$\geq 3$ & 95 & 14.3 & 2.47 & $1.89-3.19$ & $<0.0001$ & & & \\
\hline \multicolumn{9}{|c|}{ Tokyo score } \\
\hline$\leq 1$ & 198 & 67.5 & 1.00 & & & 1.00 & & \\
\hline$\geq 2$ & 227 & 23.5 & 1.99 & $1.56-2.56$ & $<0.0001$ & 1.44 & $1.11-1.88$ & 0.0058 \\
\hline \multicolumn{9}{|c|}{ CLIP score } \\
\hline 0 & 269 & 48.4 & 1.00 & & & & & \\
\hline$\geq 1$ & 156 & 22.1 & 1.48 & $1.16-1.89$ & 0.0016 & & & \\
\hline \multicolumn{9}{|c|}{ BCLC staging system } \\
\hline $0, \mathrm{~A}$ & 317 & 48.4 & 1.00 & & & & & \\
\hline $\mathrm{B}, \mathrm{C}$ & 108 & 17.7 & 2.36 & $1.83-3.04$ & $<0.0001$ & & & \\
\hline
\end{tabular}

B, Overall survival

\begin{tabular}{|c|c|c|c|c|c|c|c|c|}
\hline \multirow[b]{2}{*}{ Model } & \multirow[b]{2}{*}{$\mathrm{n}$} & \multirow[b]{2}{*}{ MST (month) } & \multicolumn{3}{|c|}{ Univariate } & \multicolumn{3}{|c|}{ Multivariate } \\
\hline & & & HR & $95 \% \mathrm{CI}$ & P-value & HR & $95 \% \mathrm{CI}$ & P-value \\
\hline \multicolumn{9}{|c|}{ REACH score } \\
\hline$\leq 2$ & 209 & 166.2 & 1.00 & & & 1.00 & & \\
\hline$\geq 3$ & 216 & 69.5 & 3.03 & $2.18-4.26$ & $<0.0001$ & 2.16 & $1.48-3.18$ & $<0.0001$ \\
\hline \multicolumn{9}{|c|}{ JIS score } \\
\hline$\leq 2$ & 361 & 116.7 & 1.00 & & & & & \\
\hline$\geq 3$ & 64 & 40.7 & 2.18 & $1.50-3.11$ & $<0.0001$ & & & \\
\hline \multicolumn{9}{|c|}{ Modified JIS score } \\
\hline$\leq 2$ & 330 & 123.4 & 1.00 & & & & & \\
\hline$\geq 3$ & 95 & 62.5 & 2.14 & $1.53-2.97$ & $<0.0001$ & & & \\
\hline \multicolumn{9}{|c|}{ Tokyo score } \\
\hline$\leq 1$ & 198 & 190.4 & 1.00 & & & 1.00 & & \\
\hline$\geq 2$ & 227 & 74.4 & 2.72 & $1.95-3.86$ & $<0.0001$ & 1.96 & $1.37-2.85$ & 0.0002 \\
\hline \multicolumn{9}{|c|}{ CLIP score } \\
\hline 0 & 269 & 116.7 & 1.00 & & & & & \\
\hline$\geq 1$ & 156 & 81.2 & 1.61 & $1.18-2.19$ & 0.0031 & & & \\
\hline \multicolumn{9}{|c|}{ BCLC score } \\
\hline $0, \mathrm{~A}$ & 317 & 123.4 & 1.00 & & & & & \\
\hline $\mathrm{B}, \mathrm{C}$ & 108 & 69.5 & 2.12 & $1.53-2.91$ & $<0.0001$ & & & \\
\hline
\end{tabular}

BCLC, Barcelona Clinic Liver Cancer; CI, confidence interval; CLIP, Cancer of the Liver Italian Program; HR, hazard ratio; JIS, Japan Integrated Staging; MST, median survival time; REACH, recurrence after curative hepatectomy. 
factors is unknown. External validation in other countries will be required.

The current study demonstrated the clinical benefits of the REACH score for evaluating the recurrence of $\mathrm{HCC}$ in patients who have undergone surgical resection. From the standpoint of everyday clinical use, a predictive system must enable the accurate detection of patient risk for early recurrence. In addition, the system should be simple and should use data that is easily obtained in daily practice. The REACH score, based on the NxS factor and clinicopathological parameters, can be used to classify HCC patients into high and low risk of recurrence following hepatic resection.

\section{References}

1. Henderson JM, Sherman M, Tavill A, Abecassis M, Chejfec G and Gramlich T: AHPBA/AJCC consensus conference on staging of hepatocellular carcinoma: Consensus statement. HPB (Oxford) 5: 243-250, 2003.

2. Forner A, Llovet JM and Bruix J: Hepatocellular carcinoma. Lancet 379: 1245-1255, 2012.

3. Llovet JM, Burroughs A and Bruix J: Hepatocellular carcinoma. Lancet 362: 1907-1917, 2003.

4. Llovet JM, Ricci S, Mazzaferro V, Hilgard P, Gane E, Blanc JF, de Oliveira AC, Santoro A, Raoul JL, Forner A, et al: Sorafenib in advanced hepatocellular carcinoma. N Engl J Med 359: 378-390, 2008

5. Gao M, Nettles RE, Belema M, Snyder LB, Nguyen VN, Fridell RA, Serrano-Wu MH, Langley DR, Sun JH, O'Boyle DR II, et al: Chemical genetics strategy identifies an HCV NS5A inhibitor with a potent clinical effect. Nature 465: 96-100, 2010.

6. Kudo M, Chung $\mathrm{H}$ and Osaki Y: Prognostic staging system for hepatocellular carcinoma (CLIP score): Its value and limitations and a proposal for a new staging system, the Japan integrated staging score (JIS score). J Gastroenterol 38: 207-215, 2003 .

7. Kudo M, Chung H, Haji S, Osaki Y, Oka H, Seki T, Kasugai H, Sasaki Y and Matsunaga T: Validation of a new prognostic staging system for hepatocellular carcinoma: The JIS score compared with the CLIP score. Hepatology 40: 1396-1405, 2004.

8. Nanashima A, Sumida Y, Morino S, Yamaguchi H, Tanaka K, Shibasaki S, Ide N, Sawai T, Yasutake T, Nakagoe T and Nagayasu T: The Japanese integrated staging score using liver damage grade for hepatocellular carcinoma in patients after hepatectomy. Eur J Surg Oncol 30: 765-770, 2004.

9. Nanashima A, Sumida Y, Abo T, Shindou H, Fukuoka H, Takeshita H, Hidaka S, Tanaka K, Sawai T, Yasutake T, et al: Modified Japan Integrated Staging is currently the best available staging system for hepatocellular carcinoma patients who have undergone hepatectomy. J Gastroenterol 41: 250-256, 2006.

10. A new prognostic system for hepatocellular carcinoma: A retrospective study of 435 patients: The cancer of the liver Italian program (CLIP) investigators. Hepatology 28: 751-755, 1998.

11. Prospective validation of the CLIP score: A new prognostic system for patients with cirrhosis and hepatocellular carcinoma. The Cancer of the Liver Italian Program (CLIP) investigators. Hepatology 31: 840-845, 2000.

12. Tateishi R, Yoshida H, Shiina S, Imamura H, Hasegawa $K$, Teratani T, Obi S, Sato S, Koike Y, Fujishima T, et al: Proposal of a new prognostic model for hepatocellular carcinoma: an analysis of 403 patients. Gut 54: 419-425, 2005.

13. Llovet JM, Brú C and Bruix J: Prognosis of hepatocellular carcinoma: The BCLC staging classification. Semin Liver Dis 19: 329-338, 1999.

14. Llovet JM, Fuster J and Bruix J: The Barcelona approach: Diagnosis, staging, and treatment of hepatocellular carcinoma. Liver Transpl 10 (2 Suppl 1): S115-S120, 2004.

15. Poon RT, Fan ST, Ng IO, Lo CM, Liu CL and Wong J: Different risk factors and prognosis for early and late intrahepatic recurrence after resection of hepatocellular carcinoma. Cancer 89: 500-507, 2000
16. Sumie S, Kuromatsu R, Okuda K, Ando E, Takata A, Fukushima N, Watanabe Y, Kojiro M and Sata M: Microvascular invasion in patients with hepatocellular carcinoma and its predictable clinicopathological factors. Ann Surg Oncol 15: 1375-1382, 2008.

17. Kim H, Park MS, Park YN, Kim H, Kim KS, Choi JS, Ahn SH, Han KH, Kim MJ and Kim KW: Preoperative radiologic and postoperative pathologic risk factors for early intra-hepatic recurrence in hepatocellular carcinoma patients who underwent curative resection. Yonsei Med J 50: 789-795, 2009.

18. Park YK, Kim BW, Wang HJ and Kim MW: Hepatic resection for hepatocellular carcinoma meeting Milan criteria in Child-Turcotte-Pugh class a patients with cirrhosis. Transplant Proc 41: 1691-1697, 2009.

19. Fan ST, Poon RT, Yeung C, Lam CM, Lo CM, Yuen WK, Ng KK, Liu CL and Chan SC: Outcome after partial hepatectomy for hepatocellular cancer within the Milan criteria. Br J Surg 98: 1292-1300, 2011.

20. Tokumitsu Y, Tamesa T, Matsukuma S, Hashimoto N, Maeda Y, Tokuhisa Y, Sakamoto K, Ueno T, Hazama S, Ogihara H, et al: An accurate prognostic staging system for hepatocellular carcinoma patients after curative hepatectomy. Int J Oncol 46: 944-952, 2015.

21. Tokumitsu Y and Nagano H: Current HCC Staging Systems: Their Uses and Limitations. In: Hepatocellular Carcinoma: Diagnosis and Treatment. Carr BI (ed). Springer, Switzerland, pp425-442, 2016.

22. Pugh RN, Murray-Lyon IM, Dawson JL, Pietroni MC and Williams R: Transection of the oesophagus for bleeding oesophageal varices. Br J Surg 60: 646-649, 1973.

23. The general rules for the clinical and pathological study of primary liver cancer. Kanehara, Tokyo, 2010.

24. Clinical Practice Guidelines for Hepatocellular Carcinoma-The Japan Society of Hepatology 2009 update. Hepatol Res 1 (40 Suppl): S2-S144, 2010.

25. Mazzaferro V, Regalia E, Doci R, Andreola S, Pulvirenti A, Bozzetti F, Montalto F, Ammatuna M, Morabito A and Gennari L: Liver transplantation for the treatment of small hepatocellular carcinomas in patients with cirrhosis. N Engl J Med 334: 693-699, 1996.

26. Minagawa M, Ikai I, Matsuyama Y, Yamaoka Y and Makuuchi M: Staging of hepatocellular carcinoma: Assessment of the Japanese TNM and AJCC/UICC TNM systems in a cohort of 13,772 patients in Japan. Ann Surg 245: 909-922, 2007.

27. Cho CS, Gonen M, Shia J, Kattan MW, Klimstra DS, Jarnagin WR, D'Angelica MI, Blumgart LH and DeMatteo RP: A novel prognostic nomogram is more accurate than conventional staging systems for predicting survival after resection of hepatocellular carcinoma. J Am Coll Surg 206: 281-291, 2008.

28. Li J, Liu Y, Yan Z, Wan X, Xia Y, Wang K, Liu J, Lau WY, Wu M and Shen F: A nomogram predicting pulmonary metastasis of hepatocellular carcinoma following partial hepatectomy. Br J Cancer 110: 1110-1117, 2014

29. Zou Q, Li J, Wu D, Yan Z, Wan X, Wang K, Shi L, Lau WY, Wu M and Shen F: Nomograms for pre-operative and post-operative prediction of long-term survival of patients who underwent repeat hepatectomy for recurrent hepatocellular carcinoma. Ann Surg Oncol 23: 2618-2626, 2016.

30. Ang SF, Ng ES, Li H, Ong YH, Choo SP, Ngeow J, Toh HC, Lim KH, Yap HY, Tan CK, et al: The Singapore Liver Cancer Recurrence (SLICER) score for relapse prediction in patients with surgically resected hepatocellular carcinoma. PLoS One 10: $\mathrm{e} 0128058,2015$.

31. Kaido T, Mori A, Ogura Y, Hata K, Yoshizawa A, Iida T, Yagi S and Uemoto S: Living donor liver transplantation for recurrent hepatocellular carcinoma after liver resection. Surgery 151: 55-60, 2012.

32. Iizuka N, Hamamoto $Y$ and Oka M: Predicting individual outcomes in hepatocellular carcinoma. Lancet 364: 1837-1839, 2004.

33. Iizuka N, Oka M, Yamada-Okabe H, Nishida M, Maeda Y, Mori N, Takao T, Tamesa T, Tangoku A, Tabuchi H, et al: Oligonucleotide microarray for prediction of early intrahepatic recurrence of hepatocellular carcinoma after curative resection. Lancet 361: 923-929, 2003.

34. Kurokawa Y, Matoba R, Takemasa I, Nagano H, Dono K, Nakamori S, Umeshita K, Sakon M, Ueno N, Oba S, et al: Molecular-based prediction of early recurrence in hepatocellular carcinoma. J Hepatol 41: 284-291, 2004. 
35. Srivastava S, Wong KF, Ong CW, Huak CY, Yeoh KG, Teh M, Luk JM and Salto-Tellez M: A morpho-molecular prognostic model for hepatocellular carcinoma. Br J Cancer 107: 334-339, 2012.

36. Yoshioka S, Takemasa I, Nagano H, Kittaka N, Noda T, Wada H, Kobayashi S, Marubashi S, Takeda Y, Umeshita K, et al: Molecular prediction of early recurrence after resection of hepatocellular carcinoma. Eur J Cancer 45: 881-889, 2009.

37. Adachi E, Maeda T, Matsumata T, Shirabe K, Kinukawa N, Sugimachi K and Tsuneyoshi M: Risk factors for intrahepatic recurrence in human small hepatocellular carcinoma. Gastroenterology 108: 768-775, 1995.

38. Ikeda K, Saitoh S, Tsubota A, Arase Y, Chayama K, Kumada H, Watanabe $\mathrm{G}$ and Tsurumaru M: Risk factors for tumor recurrence and prognosis after curative resection of hepatocellular carcinoma. Cancer 71: 19-25, 1993.

39. Morimoto O, Nagano H, Sakon M, Fujiwara Y, Yamada T, Nakagawa H, Miyamoto A, Kondo M, Arai I, Yamamoto T, et al: Diagnosis of intrahepatic metastasis and multicentric carcinogenesis by microsatellite loss of heterozygosity in patients with multiple and recurrent hepatocellular carcinomas. J Hepatol 39: 215-221, 2003.

40. Sakon M, Umeshita K, Nagano H, Eguchi H, Kishimoto S, Miyamoto A, Ohshima S, Dono K, Nakamori S, Gotoh M and Monden M: Clinical significance of hepatic resection in hepatocellular carcinoma: Analysis by disease-free survival curves. Arch Surg 135: 1456-1459, 2000.
41. Lau H, Man K, Fan ST, Yu WC, Lo CM and Wong J: Evaluation of preoperative hepatic function in patients with hepatocellular carcinoma undergoing hepatectomy. Br J Surg 84: 1255-1259, 1997.

42. Wakabayashi H, Ishimura K, Izuishi K, Karasawa $\mathrm{Y}$ and Maeta H: Evaluation of liver function for hepatic resection for hepatocellular carcinoma in the liver with damaged parenchyma. J Surg Res 116: 248-252, 2004.

43. Kokudo N, Hasegawa K, Akahane M, Igaki H, Izumi N, Ichida T, Uemoto S, Kaneko S, Kawasaki S, Ku Y, et al: Evidence-based Clinical Practice Guidelines for Hepatocellular Carcinoma: The Japan Society of Hepatology 2013 update (3rd JSH-HCC Guidelines). Hepatol Res 45: 123-127 2015.

44. Song P, Inagaki Y, Wang Z, Hasegawa K, Sakamoto Y, Arita J, Tang W and Kokudo N: High levels of gamma-glutamyl transferase and indocyanine green retention rate at $15 \mathrm{~min}$ as preoperative predictors of tumor recurrence in patients with hepatocellular carcinoma. Medicine (Baltimore) 94: e810, 2015.

45. Zhao H, Chen C, Fu X, Yan X, Jia W, Mao L, Jin H and, Qiu Y: Prognostic value of a novel risk classification of microvascular invasion in patients with hepatocellular carcinoma after resection. Oncotarget 8: 5474-5486, 2017.

46. Johnson PJ, Berhane S, Kagebayashi C, Satomura S, Teng M, Reeves HL, O'Beirne J, Fox R, Skowronska A, Palmer D, et al: Assessment of liver function in patients with hepatocellular carcinoma: A new evidence-based approach-the ALBI grade. J Clin Oncol 33: 550-558, 2015. 